increased risk of attention disorder. Children with more epileptiform discharges in their EEGs were more likely to have ADHD. Of patients with normal EEGs, $50 \%$ had ADHD whereas of children with $>6$ interictal spikes or S/W complexes per minute, $92 \%$ had ADHD. Learning disabilities were more common in the epilepsy group (42.5\% vs $25 \%$ in the control group, $\mathrm{P}<0.05$ ). (Bennett-Back O, Keren A, Zelnik N. Attention-deficit hyperactivity disorder in children with benign epilepsy and their siblings. Pediatr Neurol March 2011;44:187-192). (Respond: Dr Zelnik, Department of Pediatrics, Carmel Medical Center, 7 Michal Street, Haifa 34362, Israel. E-mail: zelnik@ netvision.net.il).

COMMENT. The prevalence of ADHD in school-age children with epilepsy is between 20\% (Gross-Tsur et al. 1997) and 60\% (Sherman et al. 2007). The prevalence of epileptiform EEGs in nonepileptic children evaluated for ADHD is $26 \%$, compared to 3.5-6.5\% in healthy children (Kaufmann et al. 2009; Millichap et al. 2010). The present report shows an increased risk of attention disorder in children with AED-resistant seizures and having more frequent epileptiform discharges in the EEG. ADHD is particularly common in benign epilepsy of childhood with centrotemporal spikes and frontal lobe epilepsy. The comorbidity of ADHD and epilepsy may depend on the effects of AEDs, psychosocial aspects of epilepsy, the type and localization of epileptogenic foci, or even common genetic factors.(Kaufmann R et al. 2009).

Frontal/prefrontal lobe growth retardation is reported in children with BCECTS complicated by cognitive impairments and behavioral problems compared with BCECTS patients without neuropsychiatric deficits. (Kanemura $\mathrm{H}$ et al. Brain Dev Feb 2011;33:106-113). Impaired frontal lobe growth is associated with frequent seizures and spike/wave discharges and neuropsychological problems. A small percentage of BCECTS patients may require early control of seizures to prevent frontal lobe growth retardation and associated cognitive and behavioral problems.

\title{
DEVELOPMENTAL DISORDERS
}

\section{RISK FACTORS FOR AUTISM IN TUBEROUS SCLEROSIS}

The prevalence and risk factors for autism spectrum disorders (ASD) in a cohort of 103 patients with tuberous sclerosis complex (TSC) were determined in a study at the Massachusetts General Hospital, Boston, MA. ASD was diagnosed in $40 \%$ of patients with TSC. Patients with ASD were younger than those without ASD (9.9 vs 16.2 years, $\mathrm{p}<0.001$ ), and had lower IQs (51 vs $81, \mathrm{p}<0.001$ ). Frequency of male gender was not significantly different ( $49 \%$ vs $42 \%$ ) in patients with and without ASD, and skin, renal, cardiac, pulmonary, and ophthalmological manifestations were similar. TSC/ASD patients were less likely to have mutations in the TSC1 gene. They had an earlier onset of seizures $(0.7$ vs 2.9 years, $\mathrm{p}<0.002)$ and more frequent seizures, and a significantly greater amount of interictal epileptiform features, especially in the left temporal lobe ( $82 \%$ vs $61 \%, \mathrm{p}<0.049)$. Infantile spasms occurred in $59 \%$ of TSC with ASD patients and $32 \%$ of TSC without ASD $(\mathrm{p}<0.014)$. On MRI, the regional distribution of tuber burden was unaltered, but patients with TSC2 and ASD had a higher prevalence of cyst-like tubers. (Numis AL, Major P, Montenegro MA, Muzykewicz DA, Pulsifer MB, Thiele EA. Identification of risk factors for autism spectrum disorder in tuberous sclerosis 
complex. Neurology March 15 2011;76:981-987) (Respond and Reprints: Dr Elizabeth A Thiele, Herscot Center for Tuberous Sclerosis Complex, 175 Cambridge Street, Suite 340, Boston, MA 02114. E-mail: ethiele@partners.org).

COMMENT. Autistic spectrum disorders are characterized by impaired social interaction, restricted interests, and repetitive behaviors. Risk of ASD development in children with TSC is increased with early persistent seizure activity in the left temporal lobe, the brain region responsible for social perception and communication. Interictal epileptiform discharges in the temporal lobe, and cyst-like tubers on MRI may provide markers for ASD in TSC.

Commonly missed signs of TSC, and presenting symptoms and signs according to age group are reported from the Massachusetts General Hospital study of 243 patients with TSC (Staley BA et al. Pediatrics Jan 2011;127:e117-e125). Average age at diagnosis was 7.5 years, $81 \%$ before age 10 years (range, birth to 73 years). The most common presenting features were new onset seizures, infantile spasms, family history of TSC, cardiac rhabdomyomas, and hypopigmented macules. Missed symptoms and signs, most commonly seizures (including infantile spasms) and dermatological signs, were reported in $39 \%$ of patients. Patients with a TSC2 genetic mutation were diagnosed on average 9 years before patients with a TSC1 mutation, and were more likely to present with infantile spasms, developmental delay, or angiofibromas. Patients with a TSC1 mutation were more likely to present with a family history of TSC or hypopigmented macules. Patients with no mutation identified were more likely to present with renal angiomyolipomas. Infantile spasms are a risk factor for slow cognitive development in TSC. Immediate diagnosis of infantile spasms by EEG hypsarrhythmia and early treatment are required to reduce seizure frequency and risk of cognitive impairment. Vigabatrin is recommended for infantile spasms secondary to TSC.

\section{MOVEMENT DISORDERS}

\section{METABOLIC BRAIN NETWORKS IN TOURETTE SYNDROME}

Researchers at the Feinstein Institute for Medical Research, Manhașset, NY, studied metabolic brain networks associated with Tourette syndrome (TS) and comorbid obsessive compulsive disorder (OCD) using PET imaging and spatial covariance analysis in 12 unmedicated patients and 12 age-matched controls. An abnormal TS-related spatial covariance pattern was characterized by reduced resting metabolic activity of the striatum and orbitofrontal cortex associated with relative increases in premotor cortex and cerebellum. In TS/OCD patients, a second metabolic pattern correlated with OCD and was characterized by reduced activity of the anterior cingulate and dorsolateral prefrontal cortical regions associated with relative increases in primary motor cortex and precuneus. The OCD pattern in individual subjects was correlated with severity of OCD. Different clinical manifestations of TS are associated with 2 distinct abnormal metabolic brain networks of potential value as biomarkers for assessing response to therapy. (Pourfar M, Feigin A, Tang CC et al. Abnormal metabolic brain networks in Tourette syndrome. Neurology March 15, 2011;76:944-952). (Respond and reprints: Dr Andrew Feigin, 